International Journal of Recent advances in Physics (IJRAP) Vol.4, No.4, November 2015

\title{
BOUND STATE SOLUTION TO SCHRODINGER EQUATION WITH MODIFIED HYLLERAAS PLUS INVERSELY QUADRATIC POTENTIAL USING SUPERSYMMETRIC QUANTUM MECHANICS APPROACH
}

\author{
Ituen .B.Okon ${ }^{1}$, Oyebola Popoola ${ }^{2}$ and Eno.E. Ituen ${ }^{1}$ \\ ${ }^{1}$ Department of Physics, University of Uyo, Uyo, Nigeria. \\ ${ }^{2}$ Department of Physics, University of Ibadan, Ibadan, Nigeria. \\ ${ }^{1}$ Department of Physics, University of Uyo, Uyo, Nigeria.
}

\begin{abstract}
In this work, we obtained an approximate bound state solution to Schrodinger equation with modified Hylleraass plus inversely quadratic potential using Supersymmetric quantum mechanics approach. Applying perkeris approximation to the centrifugal term, we obtained the eigen-energy and the normalized wave function using Gauss and confluent hypergeometric functions. We implement Fortran algorithm to obtained the numerical result of the energy for the screening parameter $\alpha=0.1,0.2,0.3,0.4$ and 0.5 . The result shows that the energy increases with an increase in the quantum state. The energy spectrum shows increase in angular quantum state spacing as the screening parameter increases.
\end{abstract}

\section{KEYWORDS}

Schrodinger, Supersymmetric Quantum Mechanics Approach, Modified Hylleraass plus Inversely Quadratic potential.

\section{INTRODUCTION}

Schrodinger wave equation belongs to non-relativistic wave equation. The total wave function of any quantum mechanical system basically provides implicitly the relevant information about the physical behavior of the system. Bound state solutions most time provides negative energies because oftenly, the energy of the particle is less than the maximum potential energy therefore, causing the particle to be trapped within the potential well. However, in a well that is infinitely long, the particles can have positive energies and are still trapped within the potential well, hence we can conclude that for infinitely long potential well, bound state energy of a particle is either less than the potential at negative infinity $(E<-\infty)$ or less than the potential at positive infinity $(E<\infty)$ which provides the reason for obtaining both negative and positive bound state energies predominantly in Klein-Gordon equation. A lot of authors developed interest in studying bound state solutions majorly due to its scientific applications in both physical and chemical sciences in particle, high energy Physics and molecular dynamics . [1-3].

DOI : 10.14810/ijrap.2015.4403 
Different analytical techniques have been adopted by different authors in providing solutions to relativistic and non- relativistic wave equations. These are: Nikiforov-Uvarov method, exact quantisation, asymptotic iteration method, supersymmetric quantum mechanics approach, factorization method, tridiagonalisation method etc.[4-10]. Some of the potentials under consideration are: woods-Saxon plus modified exponential coulomb potential, Hulthen plus generalized exponential coulomb potential, Rosen-Morse, Hulthen, pseudo harmonic, PoschlTeller, kratzer fues and Mie-Type potential, Eckart potential and P-T symmetric Hulthen potential etc [11-20]. This paper is organized as follows: section 1 is the introduction. In section 2, we introduced the concept of supersymmetric quantum mechanics approach. In section 3, we apply the concept of supersymmetry to provide the solution to Schrodinger equation using the proposed potential and obtained the energy eigen value and the wave function.. In section 4, we implement an algorithm to obtained numerical computation for the resulting energy.

The modified Hylleraas plus inversely quadratic potential is given by

$$
V(r)=\frac{-v_{0}}{\chi_{2}}\left(\frac{\chi_{1} e^{-2 \alpha r}}{1-e^{-2 \alpha r}}\right)+\frac{\chi_{3}}{r^{2}}
$$

Where $\chi_{3}$ is a constant, $\chi_{1}$ and $\chi_{2}$ are Hylleraas parameter. The graph of this potential against the inter-nuclear distance with various values of the screening parameter $\alpha=1.0,2.0,3.0$ and 4.0 is shown below. The chosen $\alpha$ is to enable one sees the nature of the graph which is quite different from the one for numerical computation.

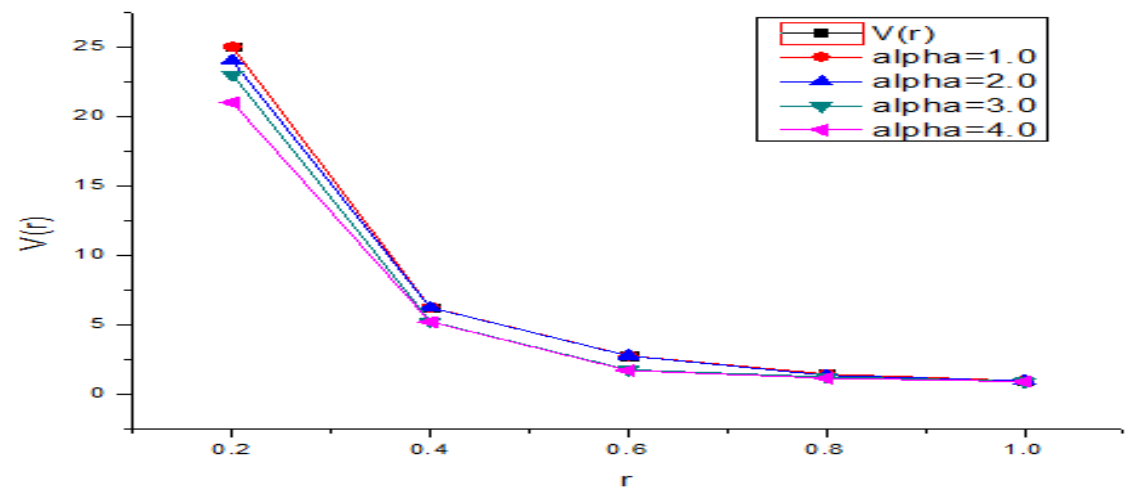

Figure a.

\section{THE CONCEPT OF SUPERYSYMMETRIC QUANTUM MECHANICS(SUSYQM)}

The supersymmetric approach deals with the partner Hamiltonian of the form

$$
H_{ \pm}=\frac{p^{2}}{2 m}+V(x)
$$

Where $\mathrm{P}$ is the momentum and $\mathrm{V}(\mathrm{x})$ is the effective potential which can be expressed in terms of super-potential as

$V_{e f f \pm}(x)=\phi^{2}(x) \pm \phi^{\prime}(x)$

The ground state energy is obtained as 
$\phi_{0}^{-1}(x)=C e^{-N}$

where $\mathrm{N}$ is the normalization constant which for very simple cases can be determined using the expression

$$
N(x)=\int_{x_{0}}^{x} \phi(r) d r
$$

However, the superpotential satisfies shape invariant condition of the form

$V_{+}\left(a_{0}, x\right)=V_{-}\left(a_{1}, x\right)+R\left(a_{1}\right)$

Where $a_{1}$ is a new set of parameter uniquely determined from the old set $a_{0}$ through the mapping $f: a_{0} \rightarrow a_{1}=f\left(a_{0}\right)$

The supersymmetric energy is determined as

$$
E_{n}=\sum_{s=1}^{n} R\left(a_{s}\right)
$$

While higher order state solutions are obtained through the expression

$$
\phi_{n}^{-}\left(a_{0}, x\right)=\prod_{s=0}^{n-1}\left(\frac{A^{\dagger}\left(a_{s}\right)}{\left(E_{n}-E_{s}\right)^{\frac{1}{2}}}\right) \phi_{0}^{-}\left(a_{n}, x\right)
$$

Where $A^{\dagger}\left(a_{s}\right)$ is a raising ladder operator expressed as

$$
A_{s}^{\dagger}=-\frac{\partial}{\partial x}+\phi\left(a_{s}, x\right)
$$

\section{RADIAL SOLUTION OF SCHRODINGER EQUATION}

Schrodinger equation is given by

$$
\frac{d^{2} R}{d r^{2}}+\left[\frac{2 \mu}{\hbar^{2}}\left(E_{n l}-V(r)\right)-\frac{l(l+1)}{r^{2}}\right] R(r)=0
$$

Substituting equation (1) into (11) gives

$$
\frac{d^{2} R}{d r^{2}}+\left[\frac{2 \mu}{\hbar^{2}}\left(E_{n l}+\frac{v_{0}}{\chi_{2}}\left(\frac{\chi_{1} e^{-2 \alpha r}}{1-e^{-2 \alpha r}}\right)-\frac{\chi_{3}}{r^{2}}\right)-\frac{l(l+1)}{r^{2}}\right] R(r)=0
$$

Let's define suitable approximation to the centrifugal term as

$$
\frac{1}{r^{2}}=\alpha^{2}\left[D_{0}+\frac{D_{1}}{\left(1-e^{-2 \alpha r}\right)}\right]
$$

Substituting equation (13) into (12) and re-arranging gives 


$$
\begin{aligned}
& \frac{d^{2} R}{d r^{2}}+\frac{1}{\left(1-e^{-2 \alpha r}\right)}\left[\frac{2 \mu v_{0} \chi_{1} e^{-2 \alpha r}}{\hbar^{2} \chi_{2}}-\frac{2 \mu v_{0} \chi_{3} \alpha^{2} D_{1}}{\hbar^{2}}-l(l+1) \alpha^{2} D_{1}\right] R(r) \\
& =-\left[\frac{2 \mu E}{\hbar^{2}}-l(l+1) \alpha^{2} D_{0}-\frac{2 \mu v_{0} \chi_{3} \alpha^{2} D_{0}}{\hbar^{2}}\right] R(r)
\end{aligned}
$$

This can also be represented as

$$
\begin{aligned}
& \frac{d^{2} R}{d r^{2}}-\frac{1}{\left(1-e^{-2 \alpha r}\right)}\left[\frac{2 \mu v_{0} \chi_{3} \alpha^{2} D_{1}}{\hbar^{2}}+l(l+1) \alpha^{2} D_{1}-\frac{2 \mu v_{0} \chi_{1} e^{-2 \alpha r}}{\hbar^{2} \chi_{2}}\right] R(r) \\
& =-\left[\frac{2 \mu E}{\hbar^{2}}-l(l+1) \alpha^{2} D_{0}-\frac{2 \mu v_{0} \chi_{3} \alpha^{2} D_{0}}{\hbar^{2}}\right] R(r)
\end{aligned}
$$

Let's define second order differential equation containing effective potential as

$$
-\frac{d^{2} R_{n l}(r)}{d r^{2}}+V_{e f f}(r) R_{n l}(r)=\tilde{E}_{n l} R_{n l}(r)
$$

In order to represent equation (15) in the form of equation (16), then equation (15) is multiply by -1 .

$$
\begin{aligned}
& -\frac{d^{2} R}{d r^{2}}+\frac{1}{\left(1-e^{-2 \alpha r}\right)}\left[\frac{2 \mu v_{0} \chi_{3} \alpha^{2} D_{1}}{\hbar^{2}}+l(l+1) \alpha^{2} D_{1}-\frac{2 \mu v_{0} \chi_{1} e^{-2 \alpha r}}{\hbar^{2} \chi_{2}}\right] R(r) \\
& =\left[\frac{2 \mu E}{\hbar^{2}}-l(l+1) \alpha^{2} D_{0}-\frac{2 \mu v_{0} \chi_{3} \alpha^{2} D_{0}}{\hbar^{2}}\right] R(r)
\end{aligned}
$$

Comparing equation (17) to (16), we obtained the following:

$$
\begin{aligned}
& V_{e f f}(r)=\frac{1}{\left(1-e^{-2 \alpha r}\right)}\left[\frac{2 \mu v_{0} \chi_{3} \alpha^{2} D_{1}}{\hbar^{2}}+l(l+1) \alpha^{2} D_{1}-\frac{2 \mu v_{0} \chi_{1} e^{-2 \alpha r}}{\hbar^{2} \chi_{2}}\right] \\
& \tilde{E}_{n l}=\left[\frac{2 \mu E}{\hbar^{2}}-l(l+1) \alpha^{2} D_{0}-\frac{2 \mu v_{0} \chi_{3} \alpha^{2} D_{0}}{\hbar^{2}}\right]
\end{aligned}
$$

Let $A=\frac{2 \mu v_{0} \chi_{3} \alpha^{2} D_{1}}{\hbar^{2}}+l(l+1) \alpha^{2} D_{1} \quad$ and $B=-\frac{2 \mu v_{0} \chi_{1}}{\hbar^{2} \chi_{2}}$

Then, the effective potential reduced to

$$
V_{\text {eff }}(r)=\frac{1}{\left(1-e^{-2 \alpha r}\right)}\left[A+B e^{-2 \alpha r}\right]
$$

The super-potential suitable for the effective potential is given as

$$
\phi(r)=\frac{-q_{1}}{\left(1-e^{-2 \alpha r}\right)}+q_{2}
$$

In order to construct partner potential, we apply equation (6) by first taking the square and first derivative of equation (21) 


$$
\begin{aligned}
& \phi^{\prime}(r)=\frac{2 \alpha q_{1} e^{-2 \alpha r}}{\left(1-e^{-2 \alpha r}\right)^{2}} \\
& \phi^{2}(r)=q_{2}^{2}-\frac{2 q_{1} q_{2}}{\left(1-e^{-2 \alpha r}\right)}+\frac{q_{1}^{2}}{\left(1-e^{-2 \alpha r}\right)^{2}}
\end{aligned}
$$

The partner potentials are

$$
\begin{aligned}
& V_{e f f}^{+}(r)=\phi^{2}(r)+\phi^{\prime}(r)=q_{2}^{2}-\frac{2 q_{1} q_{2}}{\left(1-e^{-2 \alpha r}\right)}+\frac{q_{1}^{2}}{\left(1-e^{-2 \alpha r}\right)^{2}}+\frac{2 \alpha q_{2} e^{-2 \alpha r}}{\left(1-e^{-2 \alpha r}\right)^{2}} \\
& V_{e f f}^{-}(r)=\phi^{2}(r)-\phi^{\prime}(r)=q_{2}^{2}-\frac{2 q_{1} q_{2}}{\left(1-e^{-2 \alpha r}\right)}+\frac{q_{1}^{2}}{\left(1-e^{-2 \alpha r}\right)^{2}}-\frac{2 \alpha q_{2} e^{-2 \alpha r}}{\left(1-e^{-2 \alpha r}\right)^{2}}
\end{aligned}
$$

Equation (24) and (25) satisfies shape invariant condition.

\subsection{CALCULATION OF GROUND STATE ENERGY}

The ground state energy can be calculated by solving associated Riccati equation. This equation is given as

$$
\phi^{2}(r)-\phi^{\prime}(r)=V_{e f f}(r)-\tilde{E}_{0 l}
$$

Where $\tilde{E}_{0 l}$ is the ground state energy.

Substituting equation (20) and (25) into (26) and simplifying in decreasing order of exponent gives rise to three pairs of simultaneous equations

$$
\begin{aligned}
& A+\tilde{E}_{0 l}=q_{1}^{2}+q_{2}^{2}-2 q_{1} q_{2} \\
& B-A-2 \tilde{E}_{0 l}=-2 q_{2}^{2}+2 q_{1} q_{2}-2 \alpha q_{1} \\
& \tilde{E}_{0 l}-B=q_{2}^{2}
\end{aligned}
$$

Solving the following simultaneous equations then

$$
\begin{gathered}
q_{1}=q_{2} \pm \sqrt{q_{2}^{2}+(A+B)} \\
q_{2}=-\left[\frac{A+B+2 \alpha q_{1}}{2 q_{1}}\right] \Rightarrow q_{2}^{2}=\left[\frac{A+B+2 \alpha q_{1}}{2 q_{1}}\right]^{2}
\end{gathered}
$$

The ground state energy is calculated using equation (29)

$$
\tilde{E}_{0 l}=\left[\frac{A+B+2 \alpha q_{1}}{2 q_{1}}\right]^{2}+B \Rightarrow-\tilde{E}_{0 l}=-\left[\frac{A+B+2 \alpha q_{1}}{2 q_{1}}\right]^{2}-B
$$




\subsection{CALULATION OF HIGHER ORDER SUPERSYMMETRIC ENERGY}

Using the condition of shape invariant, higher order supersymmetric energy can be calculated as using

$\bar{E}_{n l}=\sum_{k=1}^{\infty} R\left(a_{k}^{-}\right)$

Equation (33) satisfying shape invariant condition can be evaluated as follows

$R\left(a_{1}\right)=R\left(a_{0}\right)-R\left(a_{1}\right)$

$R\left(a_{2}\right)=R\left(a_{0}\right)-R\left(a_{2}\right)$

$R\left(a_{3}\right)=R\left(a_{0}\right)-R\left(a_{3}\right)$

$R\left(a_{n}\right)=R\left(a_{n-1}\right)-R\left(a_{n}\right)$

Thus, there is one to one corresponding mapping as shown below.

$q_{1}: \mapsto q_{1}+\alpha$
$a_{0}: \mapsto a_{0}+\alpha$
$a_{n}: \mapsto a_{n}+\alpha n$
$\Rightarrow q_{1}: \mapsto a_{0}$
$\Rightarrow q_{1}: \mapsto a_{n}$

Therefore, using (35), equation (31) can be written as

$q_{2}^{2}=\left[\frac{A+B+2 \alpha q_{1}}{2 q_{1}}\right]^{2} \Rightarrow q_{2}^{2}=\left[\frac{A+B+2 \alpha q_{0}}{2 q_{0}}\right]^{2}$

Using equation (34), the following evaluations is carried out

$$
\begin{aligned}
& R\left(a_{1}\right)=\left[\frac{A+B+2 \alpha q_{0}}{2 q_{0}}\right]^{2}-\left[\frac{A+B+2 \alpha q_{1}}{2 q_{1}}\right]^{2} \\
& R\left(a_{2}\right)=\left[\frac{A+B+2 \alpha q_{1}}{2 q_{1}}\right]^{2}-\left[\frac{A+B+2 \alpha q_{2}}{2 q_{2}}\right]^{2} \\
& R\left(a_{3}\right)=\left[\frac{A+B+2 \alpha q_{2}}{2 q_{2}}\right]^{2}-\left[\frac{A+B+2 \alpha q_{3}}{2 q_{3}}\right]^{2} \\
& \bar{E}_{n l}=\sum_{k=1}^{\infty} R\left(a_{k}^{-}\right)=\left[\frac{A+B+2 \alpha a_{0}}{2 a_{0}}\right]^{2}-\left[\frac{A+B+2 \alpha a_{n}}{2 a_{n}}\right]^{2}
\end{aligned}
$$




\subsection{CALCULATION OF TOTAL ENERGY}

The total energy is the sum of the ground state energy and higher order supersymmetric energy. This is given as

$$
\tilde{E}_{n l}=\bar{E}_{n l}+\tilde{E}_{0 l}
$$

Sometimes, the ground state energy can be negative (bound State condition) like in hydrogen atom which is about $-13.6 \mathrm{eV}$.

Hence, equation (38) can in this manner be expressed as

$$
\tilde{E}_{n l}=\bar{E}_{n l}+\left(-\tilde{E}_{0 l}\right)
$$

Substituting equation (32) and (37) into (39) gives

$$
\tilde{E}_{n l}=-\left\{\left[\frac{A+B+2 \alpha a_{n}}{2 a_{n}}\right]+B\right\}
$$

Let's recall equation (30)

$$
q_{1}=q_{2} \pm \sqrt{q_{2}^{2}+(A+B)} \Rightarrow q_{1}=\alpha \pm \sqrt{\alpha^{2}+(A+B)} \quad, \quad q_{2}: \mapsto \alpha
$$

If $a_{n}: \mapsto a_{n}+\alpha n, \quad$ for $\quad q_{1}: \mapsto a_{n}$

Then $a_{n}=\alpha n+\alpha \pm \sqrt{\alpha^{2}+(A+B)} \Rightarrow a_{n}=\alpha\left[(n+1) \pm \sqrt{\alpha^{2}+(A+B)}\right]$

Substituting equation (18b) and equation (41) into (40) and simplifying gives the total energy as

$$
\begin{aligned}
& E_{n l}=-\frac{\hbar^{2}}{2 \mu}\left\{\left[\frac{\left(\frac{2 \mu \chi_{3} \alpha^{2} D_{1}}{\hbar^{2}}+l(l+1) \alpha^{2} D_{1}-\frac{2 \mu v_{0} \chi_{1}}{\chi_{2}}\right)+2 \alpha^{2}\left(n+1 \pm \sqrt{\frac{2 \mu \chi_{3} \alpha^{2} D_{1}}{\hbar^{2}}+l(l+1) \alpha^{2} D_{1}-\frac{2 \mu v_{0} \chi_{1}}{\hbar^{2} \chi_{2}}}\right)}{2 \alpha\left(n+1 \pm \sqrt{\frac{2 \mu \chi_{3} \alpha^{2} D_{1}}{\hbar^{2}}+l(l+1) \alpha^{2} D_{1}-\frac{2 \mu v_{0} \chi_{1}}{\hbar^{2} \chi_{2}}}\right)}\right]-\frac{2 \mu v_{0} \chi_{1}}{\hbar^{2} \chi_{2}}\right\} \\
& +\chi_{3} \alpha^{2} D_{0}+\frac{\hbar^{2} l(l+1) \alpha^{2} D_{0}}{2 \mu}
\end{aligned}
$$

Equation (42) is the energy equation for Hylleraas plus inversely quadratic potential. However, because of the plus and minus accaompanied by the square root sign, equation (42) can be expressed as follows:

$$
\begin{aligned}
& E_{n l}=-\frac{\hbar^{2}}{2 \mu}\left\{\left[\frac{\left(\frac{2 \mu \chi_{3} \alpha^{2} D_{1}}{\hbar^{2}}+l(l+1) \alpha^{2} D_{1}-\frac{2 \mu v_{0} \chi_{1}}{\chi_{2}}\right)+2 \alpha^{2}\left(n+1+\sqrt{\frac{2 \mu \chi_{3} \alpha^{2} D_{1}}{\hbar^{2}}+l(l+1) \alpha^{2} D_{1}-\frac{2 \mu v_{0} \chi_{1}}{\hbar^{2} \chi_{2}}}\right)}{2 \alpha\left(n+1+\sqrt{\frac{2 \mu \chi_{3} \alpha^{2} D_{1}}{\hbar^{2}}+l(l+1) \alpha^{2} D_{1}-\frac{2 \mu v_{0} \chi_{1}}{\hbar^{2} \chi_{2}}}\right)}\right]-\frac{2 \mu v_{0} \chi_{1}}{\hbar^{2} \chi_{2}}\right\} \\
& +\chi_{3} \alpha^{2} D_{0}+\frac{\hbar^{2} l(l+1) \alpha^{2} D_{0}}{2 \mu}
\end{aligned}
$$


International Journal of Recent advances in Physics (IJRAP) Vol.4, No.4, November 2015

$$
\begin{aligned}
& E_{n l}=-\frac{\hbar^{2}}{2 \mu}\left\{\left[\frac{\left(\frac{2 \mu \chi_{3} \alpha^{2} D_{1}}{\hbar^{2}}+l(l+1) \alpha^{2} D_{1}-\frac{2 \mu v_{0} \chi_{1}}{\chi_{2}}\right)+2 \alpha^{2}\left(n+1-\sqrt{\frac{2 \mu \chi_{3} \alpha^{2} D_{1}}{\hbar^{2}}+l(l+1) \alpha^{2} D_{1}-\frac{2 \mu v_{0} \chi_{1}}{\hbar^{2} \chi_{2}}}\right)}{2 \alpha\left(n+1-\sqrt{\frac{2 \mu \chi_{3} \alpha^{2} D_{1}}{\hbar^{2}}+l(l+1) \alpha^{2} D_{1}-\frac{2 \mu v_{0} \chi_{1}}{\hbar^{2} \chi_{2}}}\right)}\right]-\frac{2 \mu \omega_{0} \chi_{1}}{\hbar^{2} \chi_{2}}\right\} \\
& +\chi_{3} \alpha^{2} D_{0}+\frac{\hbar^{2} l(l+1) \alpha^{2} D_{0}}{2 \mu}
\end{aligned}
$$

\subsection{CALCULATION OF THE WAVE FUNCTION}

Furthermore, in order to calculate the radial wave function, we used the coordinate transformation $s=e^{-2 \alpha r}$ into equation (16) and obtained the following

$$
\frac{d^{2} R}{d r^{2}}+\frac{(1-s)}{s(1-s)} \frac{d R}{d s}+\frac{1}{s^{2}(1-s)^{2}}\left\{\begin{array}{l}
-\left(\frac{v_{0} \chi_{1}}{4 \alpha^{2} \chi_{2}}+\frac{l(l+1) D_{0}}{4}+\frac{\chi_{3} D_{0}}{4}-\frac{2 \mu E}{4 \hbar^{2} \alpha^{2}}\right) s^{2} \\
+\left(\frac{v_{0} \chi_{1}}{4 \alpha^{2} \chi_{2}}+\frac{2 \chi_{3} D_{0}}{4}+\frac{\chi_{3} D_{1}}{4}+\frac{2 l(l+1) D_{0}}{4}+\frac{l(l+1) D_{1}}{4}-\frac{\mu E}{\hbar^{2} \alpha^{2}}\right) s \\
-\left(\frac{\chi_{3} D_{0}}{4}+\frac{\chi_{3} D_{1}}{4}+\frac{l(l+1) D_{0}}{4}+\frac{l(l+1) D_{1}}{4}-\frac{2 \mu E}{4 \hbar^{2} \alpha^{2}}\right)
\end{array}\right.
$$

The corresponding radial wave function is then given by

$$
\begin{aligned}
& R_{n l}(r)=N_{n}\left(e^{-2 \alpha r}\right)^{\sqrt{\frac{\chi_{3} D_{0}}{4}+\frac{\chi_{3} D_{1}}{4}+\frac{l(l+1) D_{0}}{4}+\frac{l(l+1) D_{1}}{4}-\frac{2 \mu E}{4 \hbar^{2} \alpha^{2}}}}\left(1-e^{-2 \alpha r}\right)^{-1-\sqrt{\frac{\chi_{3} D_{0}}{4}+\frac{\chi_{3} D_{1}}{4}+\frac{l(l+1) D_{0}}{4}+\frac{l(l+1) D_{1}}{4}-\frac{2 \mu E}{4 \hbar^{2} \alpha^{2}}}} \\
& \times P_{n}^{\left[\left(1+2 \sqrt{\frac{\chi_{3} D_{0}}{4}+\frac{\chi_{3} D_{1}}{4}+\frac{l(l+1) D_{0}}{4}+\frac{l(l+1) D_{1}}{4}-\frac{2 \mu E}{4 \hbar^{2} \alpha^{2}}}\right) \cdot\left(3+2 \sqrt{\frac{\chi_{3} D_{0}}{4}+\frac{\chi_{3} D_{1}}{4}+\frac{l(l+1) D_{0}}{4}+\frac{l(l+1) D_{1}}{4}-\frac{2 \mu E}{4 \hbar^{2} \alpha^{2}}}\right)\right]}\left(1-2 e^{-2 \alpha r}\right)
\end{aligned}
$$

\section{NUMERICAL COMPUTATION OF THE ENERGY EQUATION}

Using equation (43), we implement Fortran algorithm to compute for the energy of the equation in electron volt with various values of the screening parameter $\alpha$. We use the following values for our computation. $\quad V_{0}=\hbar=\mu=1.0, \quad D_{0}=\frac{1}{12}, D_{1}=1.0, \chi_{1}=-1, \chi_{2}=2, \chi_{2}=1$. 
International Journal of Recent advances in Physics (IJRAP) Vol.4, No.4, November 2015

Bound State energy with $\alpha=0.1$ for different quantum state

\begin{tabular}{|c|c|c|c|c|c|c|c|c|c|c|c|}
\hline$n$ & $l$ & $E_{n}(\alpha=0.1) e V$ & $n$ & $l$ & $E_{n}(\alpha=0.1) \mathrm{eV}$ & $n$ & $l$ & $E_{n}(\alpha=0.1) e V$ & $n$ & $l$ & $E_{n}(\alpha=0.1) e V$ \\
\hline 0 & $\mathbf{0}$ & -1.81557810 & 0 & 1 & -1.83414400 & 0 & 2 & -1.87091950 & 0 & 3 & -1.92523000 \\
\hline 1 & 0 & -1.39580210 & 1 & 1 & -1.40959080 & 1 & 2 & -1.43698190 & 1 & 3 & -1.47761940 \\
\hline 2 & 0 & -1.18513580 & 2 & 1 & -1.19601170 & 2 & 2 & -1.21765080 & 2 & 3 & -1.24983630 \\
\hline 3 & 0 & -1.05848570 & 3 & 1 & -1.06744430 & 3 & 2 & -1.08528610 & 3 & 3 & -1.11186640 \\
\hline 4 & 0 & -0.97394794 & 4 & 1 & -0.98155713 & 4 & 2 & -0.99672174 & 4 & 3 & -1.01933810 \\
\hline 5 & 0 & -0.91351247 & 5 & 1 & -0.92012280 & 5 & 2 & -0.93330324 & 5 & 3 & -0.95297605 \\
\hline 6 & 0 & -0.86815786 & 6 & 1 & -0.87399983 & 6 & 2 & -0.88565266 & 6 & 3 & -0.90305570 \\
\hline 7 & 0 & -0.83286536 & 7 & 1 & -0.83809840 & 7 & 2 & -0.84853950 & 7 & 3 & -0.86414030 \\
\hline 8 & 0 & -0.80462086 & 8 & 1 & -0.80935950 & 8 & 2 & -0.81881640 & 8 & 3 & -0.83295200 \\
\hline
\end{tabular}

Table 1

Bound State energy with $\alpha=0.2$ for different quantum state

\begin{tabular}{|c|c|c|c|c|c|c|c|c|c|c|}
\hline$n$ & $l$ & $E_{n}(\alpha=0.2) e V$ & $n$ & $l$ & $E_{n}(\alpha=0.2) e V$ & & $l$ & $E_{n}(\alpha=0.2) e V$ & $n l$ & $E_{n}(\alpha=0.2) \mathrm{eV}$ \\
\hline 0 & 0 & -1.25588080 & 0 & 1 & -1.29197690 & 0 & 2 & -1.36170600 & \begin{tabular}{l|l}
0 & 3
\end{tabular} & -1.46096090 \\
\hline 1 & 0 & -1.04142170 & 1 & 1 & -1.06843020 & 1 & 2 & -1.12113100 & \begin{tabular}{l|l}
1 & 3 \\
\end{tabular} & -1.19725980 \\
\hline 2 & 0 & -0.93265150 & 2 & 1 & -0.95405185 & 2 & 2 & -0.99604530 & \begin{tabular}{l|l}
2 & 3 \\
\end{tabular} & -1.05721940 \\
\hline 3 & 0 & -0.86688805 & 3 & 1 & -0.88456786 & 3 & 2 & -0.91938436 & \begin{tabular}{l|l}
3 & 3 \\
\end{tabular} & -0.97037660 \\
\hline 4 & 0 & -0.82283480 & 4 & 1 & -0.83788250 & 4 & 2 & -0.86758830 & \begin{tabular}{l|l}
4 & 3
\end{tabular} & -0.91125740 \\
\hline 5 & 0 & -0.79126420 & 5 & 1 & -0.80435646 & 5 & 2 & -0.83024790 & \begin{tabular}{l|l}
5 & 3 \\
\end{tabular} & -0.86841345 \\
\hline 6 & 0 & -0.76752913 & 6 & 1 & -0.77911305 & 6 & 2 & -0.80205260 & \begin{tabular}{l|l}
6 & 3 \\
\end{tabular} & -0.83593720 \\
\hline 7 & 0 & -0.74903460 & 7 & 1 & -0.75942045 & 7 & 2 & -0.78000940 & \begin{tabular}{l|l}
7 & 3 \\
\end{tabular} & -0.81047153 \\
\hline 8 & 0 & -0.73421750 & 8 & 1 & -0.74362910 & 8 & 2 & -0.76230270 & \begin{tabular}{l|l}
8 & 3
\end{tabular} & -0.78996754 \\
\hline
\end{tabular}

Table 2

Bound State energy with $\alpha=0.3$ for different quantum state

\begin{tabular}{|c|c|c|c|c|c|c|c|c|c|c|c|}
\hline$n$ & $l$ & $E_{n}(\alpha=0.3) e V$ & $n$ & $l$ & $E_{n}(\alpha=0.3) e V$ & & $l$ & $E_{n}(\alpha=0.3) e V$ & & $l$ & $E_{n}(\alpha=0.3) e V$ \\
\hline 0 & 0 & -1.11232240 & 0 & 1 & -1.16417930 & 0 & 2 & -1.26113510 & 0 & 3 & -1.39352740 \\
\hline 1 & 0 & -0.96447120 & 1 & 1 & -1.00370690 & 1 & 2 & -1.07845380 & 1 & 3 & -1.18307350 \\
\hline 2 & 0 & -0.88827163 & 2 & 1 & -0.91957426 & 2 & 2 & -0.97985350 & 2 & 3 & -1.06547440 \\
\hline 3 & 0 & -0.84179723 & 3 & 1 & -0.86777450 & 3 & 2 & -0.91814520 & 3 & 3 & -0.99038374 \\
\hline 4 & 0 & -0.81049335 & 4 & 1 & -0.83267320 & 4 & 2 & -0.87588680 & 4 & 3 & -0.93828130 \\
\hline 5 & 0 & -0.78797410 & 5 & 1 & -0.80731654 & 5 & 2 & -0.84513450 & 5 & 3 & -0.90001200 \\
\hline 6 & 0 & -0.77099670 & 6 & 1 & -0.78814130 & 6 & 2 & -0.82175210 & 6 & 3 & -0.87071250 \\
\hline 7 & 0 & -0.75773966 & 7 & 1 & -0.77313274 & 7 & 2 & -0.80337375 & 7 & 3 & -0.84755990 \\
\hline 8 & 0 & -0.74710070 & 8 & 1 & -0.76106584 & 8 & 2 & -0.78854835 & 8 & 3 & -0.8288036 \\
\hline
\end{tabular}

Table 3 
International Journal of Recent advances in Physics (IJRAP) Vol.4, No.4, November 2015

Bound State energy with $\alpha=0.4$ for different quantum state

\begin{tabular}{|c|c|c|c|c|c|c|c|c|c|c|c|}
\hline$n$ & $l$ & $E_{n}(\alpha=0.4) e V$ & $n$ & & $E_{n}(\alpha=0.4) e V$ & $n$ & & $E_{n}(\alpha=0.4) e$ & & $l$ & $E_{n}(\alpha=0.4) e V$ \\
\hline 0 & 0 & -1.07219960 & 0 & 1 & -1.13772730 & 0 & 2 & -1.25619530 & 0 & 3 & -1.41204810 \\
\hline 1 & 0 & -0.95648575 & 1 & 1 & -1.00673560 & 1 & 2 & -1.10005060 & 1 & 3 & -1.22680750 \\
\hline 2 & 0 & -0.89565750 & 2 & 1 & -0.93608590 & 2 & 2 & -1.01235960 & 2 & 3 & -1.11805080 \\
\hline 3 & 0 & -0.85815040 & 3 & 1 & -0.89188860 & 3 & 2 & -0.95620050 & 3 & 3 & -1.04651460 \\
\hline 4 & 0 & -0.83271027 & 4 & 1 & -0.86163010 & 4 & 2 & -0.91715777 & 4 & 3 & -0.99588360 \\
\hline 5 & 0 & -0.81432050 & 5 & 1 & -0.83961457 & 5 & 2 & -0.88844097 & 5 & 3 & -0.95816210 \\
\hline 6 & 0 & -0.80040705 & 6 & 1 & -0.82287750 & 6 & 2 & -0.86643210 & 6 & 3 & -0.92897120 \\
\hline 7 & 0 & -0.78951290 & 7 & 1 & -0.80972373 & 7 & 2 & -0.84902660 & 7 & 3 & -0.90571094 \\
\hline 8 & 0 & -0.78075130 & 8 & 1 & -0.79911387 & 8 & 2 & -0.83491695 & 8 & 3 & -0.88674080 \\
\hline
\end{tabular}

Table 4

Bound State energy with $\alpha=0.5$ for different quantum state

\begin{tabular}{|c|c|c|c|c|c|c|c|c|c|c|c|}
\hline$n$ & $l$ & $E_{n}(\alpha=0.5) e$ & $n$ & $l$ & $E_{n}(\alpha=0.5) e$ & $n$ & $l$ & $E_{n}(\alpha=0.5) e$ & & $l$ & $E_{n}(\alpha=0.5) \mathrm{eV}$ \\
\hline 0 & 0 & -1.07287570 & 0 & 1 & -1.15000000 & 0 & 2 & -1.28518370 & 0 & 3 & -1.45766960 \\
\hline 1 & 0 & -0.97570810 & 1 & 1 & -1.03571430 & 1 & 2 & -1.14444880 & 1 & 3 & -1.28834840 \\
\hline 2 & $\mathbf{0}$ & -0.92349565 & 2 & 1 & -0.97222220 & 2 & 2 & -1.06231940 & 2 & 3 & -1.18440910 \\
\hline 3 & 0 & -0.89090130 & 3 & 1 & -0.93181820 & 3 & 2 & -1.00849700 & 3 & 3 & -1.11411000 \\
\hline 4 & 0 & -0.86861694 & 4 & 1 & -0.90384614 & 4 & 2 & -0.97049820 & 4 & 3 & -1.06339450 \\
\hline 5 & 0 & -0.85241880 & 5 & 1 & -0.88333330 & 5 & 2 & -0.94223930 & 5 & 3 & -1.02507970 \\
\hline 6 & 0 & -0.84011304 & 6 & 1 & -0.86764705 & 6 & 2 & -0.92040080 & 6 & 3 & -0.99511280 \\
\hline 7 & 0 & -0.83044730 & 7 & 1 & -0.85526310 & 7 & 2 & -0.90301790 & 7 & 3 & -0.97103360 \\
\hline 8 & 0 & -0.82265410 & 8 & 1 & -0.84523810 & 8 & 2 & -0.88885320 & 8 & 3 & -0.95126210 \\
\hline
\end{tabular}

Table 5

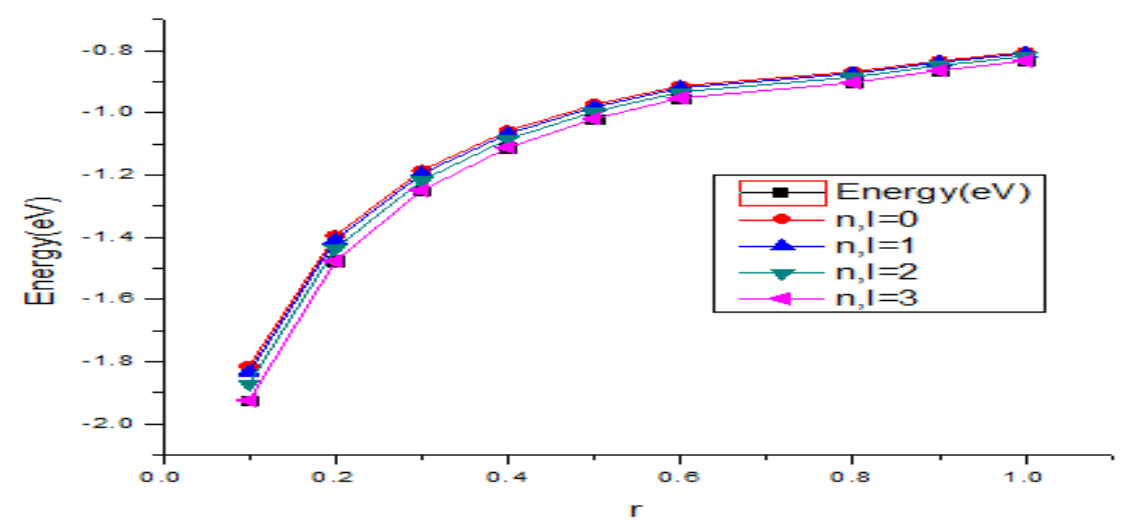

Figure 1: The graph of energy against the distance for different quantum state with $\alpha=0.1$. 
International Journal of Recent advances in Physics (IJRAP) Vol.4, No.4, November 2015

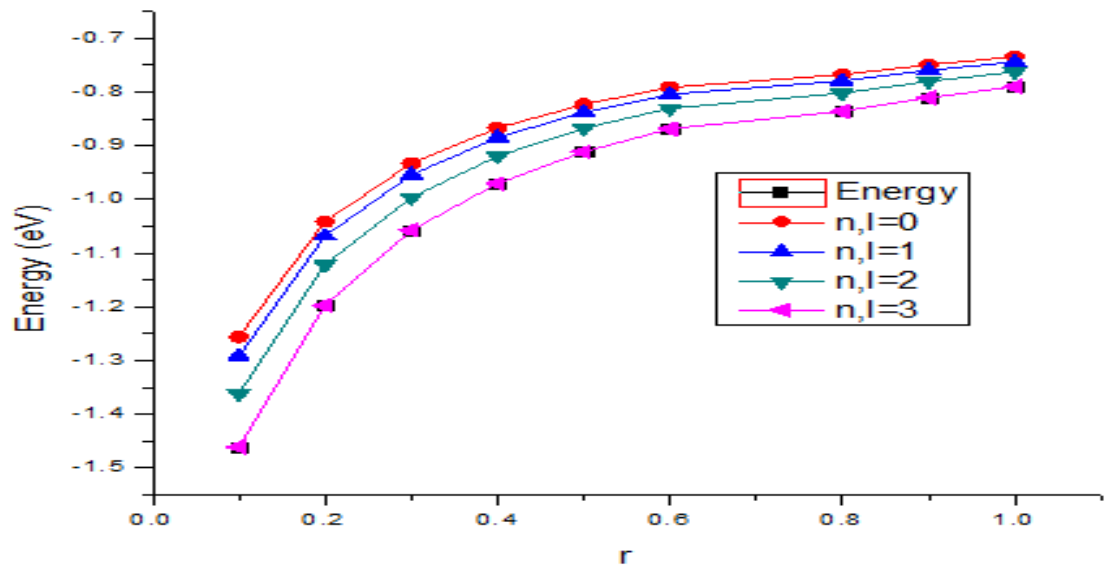

Figure 2:The graph of energy against the distance for different quantum state with $\alpha=0.2$.

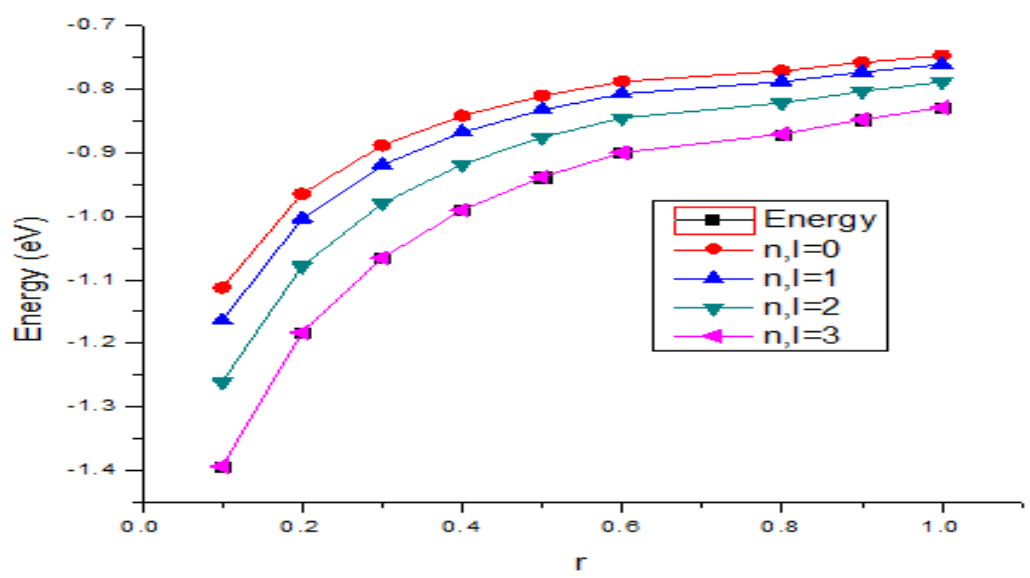

Figure 3: The graph of energy against the distance for different quantum state with $\alpha=0.3$.

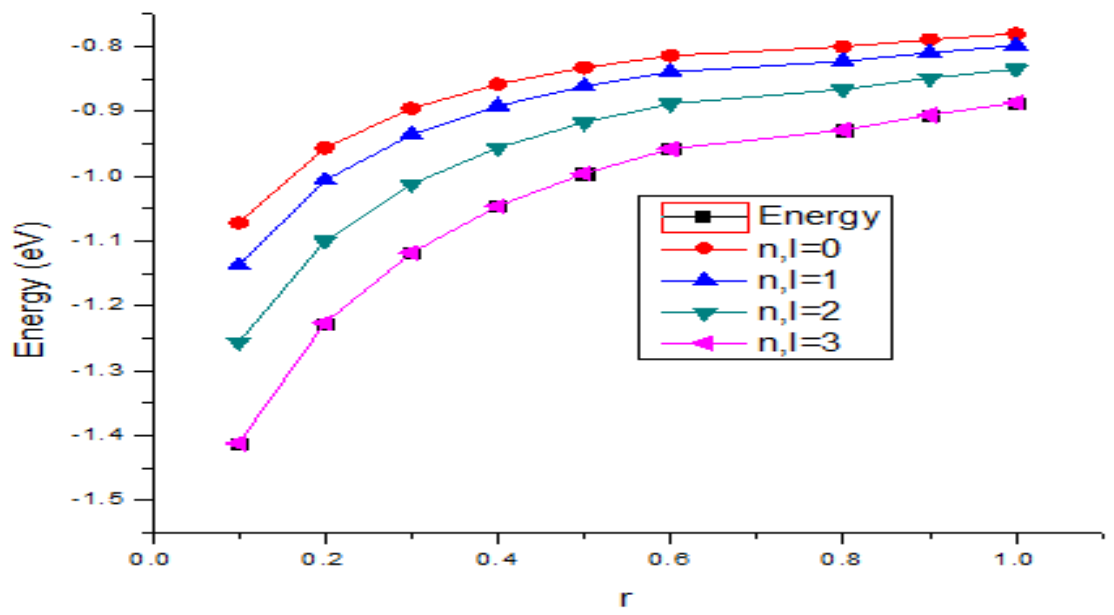

Figure 4: The graph of energy against the distance for different quantum state with $\alpha=0.4$. 


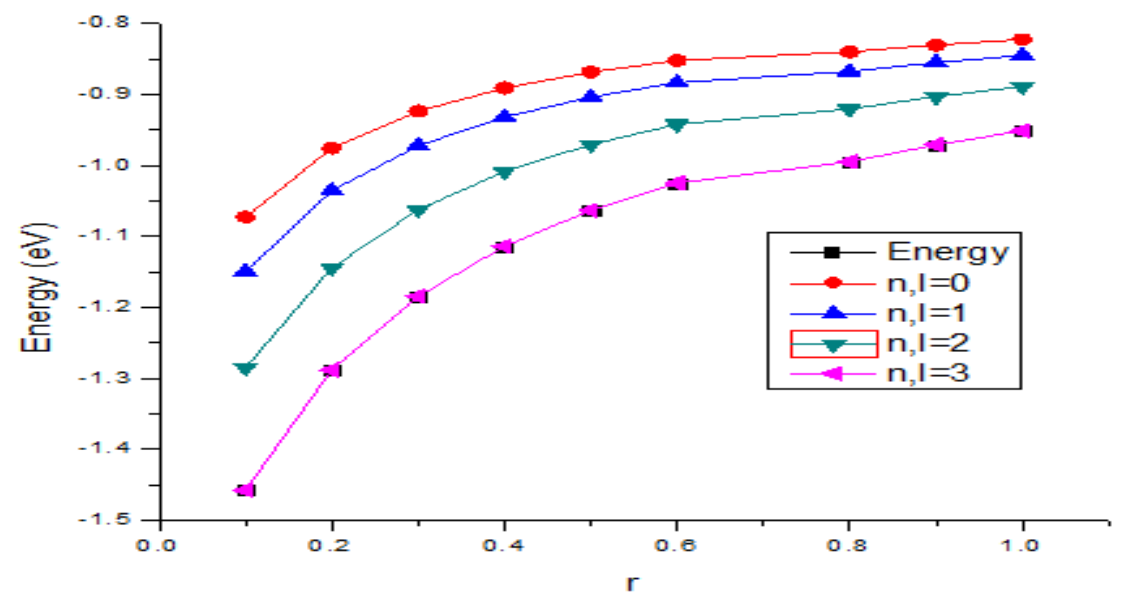

Figure 5. : The graph of energy against the distance for different quantum state with $\alpha=0.5$.

\section{CONCLUSION}

We used supersymmetric quantum mechanics approach to obtained bound state solution to Hylleraas plus inversely quadratic potential using Schrodinger equation. We also obtained eigen energy and the wave function. The result shows that the energy is negative to ascertain bound state condition and increases with an increase in quantum as shown in tables (1-5). The energy graphical spectrum shows that the interspacing within the quantum state is more pronounce the value of $\alpha$ increases. This can be shown from figures (1-5).

\section{ACKNOWLEDGEMENT}

We are deeply grateful to the referees for their useful comments which we have significantly use to improve the article.

\section{REFERENCES}

[1] OkonI.B , Ituen E.E, .Popoola O.O and Antia A.D.(2013), "Analytical Solutions of Schrodinger Equation with Mie-Type Potential Using Factorisation Method", International Journal of Recent Advances In Physics, Vol.2, No.2, pp1-7 .

[2] Okon I.B, Isonguyo C.N, Ituen, E.E and Ikot A.N. (2014), "Energy Spectrum for Some Diatomic Molecules with Generalized Manning-Rosen Potential Using Supersymmetric Quantum Mechanics (SUSY)". Conference proceedings of Nigerian Institute of Physics, 2014.

[3] Okon I. B, Popoola O.O and Isonguyo. C.N (2014), "Exact Bound state Solution of q-deformed Woods-Saxon plus modified Coulomb Potential Using Conventional Nikiforov-Uvarov Method", International Journal of Recent Advances in Physics Vol. 3, No.4.

[4] Okon I.B and Popoola. O.O.(2015), ,Bound state solution of Schrodinger equation with Hulthen plus generalised exponential coulomb potential using Nikiforov-Uvarov method", International Journal of Recent Advances in Physics. Vol.4, No.3.Doi:10.14810/ijrap.2015.4301.

[5] Isonguyo C. N, Okon, I. B and Ikot A. N (2013), "Semi-relativistic treatment of Hellmann potential using Supersymmetric Quantum Mechanics", Journal of the Nigerian Association of Mathematical Physics (NAMP Journal). Vol.25, No. 2, pp121-126. 
[6] Isonguyo, C. N , . Okon I.B, Ikot A.N and Hassanabadi H. (2014) , "Solution of Klein-Gordon Equation for some diatomic molecules with New Generalised Morse-like potential Using SUSYQM", Bull. Korean Chem. Soc. 2014, Vol. 35, No. $12 \quad 3443$ http://dx.doi.org/10.5012/bkcs.2014.35.12.3443

[7] Nikiforov A.F, Uvarov V. B (1988), " Special Functions of Mathematical Physics,", Birkhauser, Bassel

[8] Dong S.H., Factorization Method in Quantum Mechanics. (2007). Springer-Verlag, Berlin

[9] Ikhdair S. M. and Sever R.( 2007). "A perturbative treatment for the bound states of the Hellmann potential”, Journal of Molecular Structure, vol. 809, no. 1-3, pp. 103-113

[10] Berkdemir C., Berkdemir A. and Sever R. ( 2006) "Systematical approach to the exact solution of the Dirac equation for a deformed form of the Woods-Saxon potential”, Journal of Physics A, vol. 39, no. 43, pp. 13455-13463.

[11] Fl“ugge S., Practical Quantum Mechanics, Springer- Verlag, Berlin (1994).

[12] R. Dutt R., Chowdhury. K and Varshni,Y.P (1985), "An improved calculation foscreened Coulomb potentials in Rayleigh-Schrodinger”, perturbation theory. J. Phys. A: Math. Gen. vol. 18, pp13791388 .

[13] Ikhdair S. M andSever R .(2007), "An alternative simple solution of the sextic anharmonic oscillator and perturbed coulomb problems," International Journal of Modern Physics C, vol. 18, No10, pp1571.

[14] Oyewumi K.J, Akinpelu O.E and Agboola A.D (2008) "Exactly Complete Solutions of the Pseudoharmonic Potential in N-Dimensions," International Journal of Theoretical Physics, Vol. 47, No. 4, pp. 1039-1057(2008) doi:10.1007/s10773-007-9532-x.

[15] Hassanabadi H., Zarrinkamar S. and Rajabi A.A. (2011) "Exact solutions of D-dimensional schrödinger equation for an energy-dependent potential by NU method" Commun. Theor. Phys. Vol. 55 , pp 541.

[16] Ikot A.N, Antia A.D, Akpabio, L.E and Obu, A. J (2011), “Analytic Solutions of Schrodinger Equation with Two-Dimensional Harmonic Potential in Cartesian and Polar Coordinates via Nikiforov-Uvarov Method", Journal of Vectorial Relativity, Vol. 6, No. 2, pp. 65-76.

[17] Greene R. L and Aldrich C. (1976). "Variational wave functions for a screened Coulomb potential", Physical Review A, vol. 14, no. 6, pp. 2363-2366. pp1-7.

[18] Bera P.K(2012)" The exact solutions for the interaction $V(r)=\alpha r^{2 d-2}-\beta r^{d-2}$ by NikiforovUvarov method" Pramana-J. Phys Vol. 78, pp667.

[19] Ikhdair S. M. and Sever R. ( 2011). "Relativistic and nonrelativistic bound states of the isotonic oscillator by Nikiforov-Uvarov method," Journal of Mathematical Physics, vol. 52, no. 12, article 122108.

[20] Fakhri H., Sadeghi J (2004) "Supersymmetry approaches to the bound states of the generalized Woods-Saxon potential” Mod. Phys. Lett. A Vol. 19, pp615-625.

[21] Khounfais K., Boudjedaa T. and Chetouani L (2004), "Scattering matrix for Feshbach- Villars equation for spin 0 and 1/2: Woods-Saxon potential” ,Czech. J. Phys. Vol.54, pp697-792.

[22] Ikot A.N, Awoga O. A. Akpabio, L. E and Antia A. D (2011) " Exact Solutions of Schrodinger equation with Woods-Saxon Plus Rosen-Morse Potential”, Elixir vib. Spec.Vol. 2011, pp23032307. 